Mitteilungen der Österreichischen Geographischen Gesellschaft, 159. Jg. (Jahresband), Wien 2017, S. 243-263

DOI 10.23781/moegg 159-243

\title{
Wertketten in der Milchwirtschaft: Die Ausdifferenzierung Der VertriebsWege KLEINER KäSEREIEN in SLOWENIEN
}

\author{
Nicolai Dellmann und Markus Hassler, beide Marburg/Lahn* \\ Erste Einreichung / initial submission: 05/2017; revidierte Fassung / revised submission: 10/2017; \\ endgültige Annahme / final acceptance: 10/2017 \\ mit 2 Abb. und 2 Tab. im Text
}

\section{INHALT}

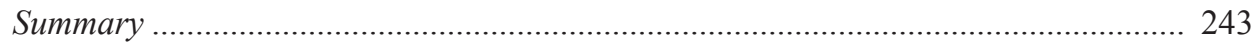

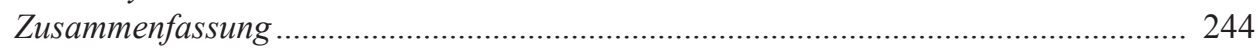

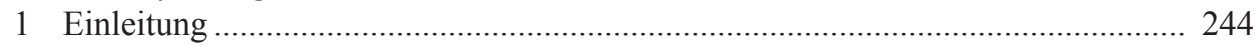

2 Theorie: Wertketten in der Milchwirtschaft ........................................................ 247

3 Die slowenische Milchwirtschaft: Struktur, Politik und Regulierung .................. 250

4 Vertriebsstrukturen in der slowenischen Milchwirtschaft .................................... 252

5 Direkte Distributionsformen ...................................................................... 255

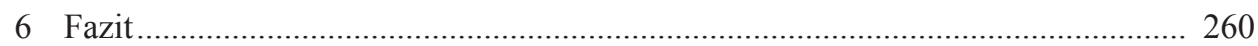

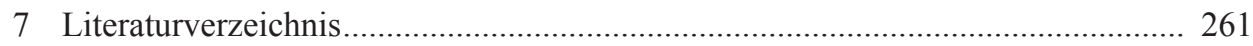

\section{Summary}

Value chains in dairy economy: differentiation processes of small cheese dairies' sales channels in Slovenia

The Slovenian dairy industry is characterised by processes of consolidation that have led to a sharp decline of small producers. Thus, Slovenian dairy farmers develop specific strategies to allow for a survival in the production process. This article analyses various sets of activities Slovenian small-scale producers develop to implement own direct sales channels as well as further processing strategies. These strategies grow more important for Slovenian dairy farmers since milk prices remain very volatile and regulatory mech-

\footnotetext{
* Dipl.-Geogr. Nicolai Dellmann, Prof. Dr. Markus Hassler, beide Philipps-Universität Marburg, Arbeitsgruppe Regionalforschung und -politik, Fachbereich Geographie, Deutschhausstraße 10, D-35032 Marburg/Lahn, Deutschland; E-Mail: nicolai.dellmann@geo, markus.hassler@geo
} 
anisms, which protect the milk farmers, erode. The article emphasises the connection between global events, such as financial crises, and the changes on the farm level. The drawback of the increasing consolidation of retail and globalisation in milk processing, together with milk prices, were identified to be the main driver mechanisms for the producers to create their own direct distribution patterns. Contemporary approaches to value chain analyses, such as the Global Value Chain and Global Production Networks, provide the theoretical framework for this research, which primarily serves to understand the power constellations in the Slovenian milk sector.

Keywords: Value chain analysis, power, distribution channels, milk sector, Slovenia, micro dairies

\section{Zusammenfassung}

Die slowenische Milchindustrie ist von Prozessen der Konsolidierung gekennzeichnet, die einen starken Rückgang kleiner Produzenten zur Folge haben. Vor diesem Hintergrund entwickeln slowenische Milchbauern besondere Strategien, um im Produktionsprozess bestehen zu können. Dieser Artikel stellt eben solche Strategien zur Ausdifferenzierung eigener, direkter Vertriebswege sowie Weiterverarbeitungsprozesse dar, die im Zuge volatiler Milchpreise und des Wegbrechens regulatorischer Mechanismen immer dringlicher auf die slowenischen Milchbauern Einfluss nehmen. Dazu wurden Veränderungen auf der Unternehmensebene der Produzenten in den Zusammenhang mit globalen Ereignissen wie Wirtschaftskrisen gestellt. Die zunehmende Konsolidierung des Einzelhandels und die Globalisierung in der Milchweiterverarbeitung konnten dabei als wichtige Antriebsmechanismen identifiziert werden, die zusammen mit dem Milchpreis die Produzenten dazu veranlassten, eigene, direkte Distributionsmuster zu schaffen. Zeitgenössische Ansätze der Wertkettenanalysen wie beispielsweise die Global Value Chain und Global Production Networks bilden den theoretischen Rahmen dieser Forschung, die primär dazu dient, die Machtkonstellationen im slowenischen Milchsektor zu verstehen.

Schlagwörter: Wertkettenanalyse, Macht, Vertriebswege, Milchsektor, Slowenien, Mikrokäsereien

\section{Einleitung}

Milch als Nahrungsmittel kommt aus einem zunehmend komplexen und sich rasch wandelnden Produktionssystem (Dries et al. 2009; Swinnen et al. 2006; Douphrate et al. 2013). Dieses System ist geprägt von der zunehmenden Bedeutung großer, wachsender Produzenten und einer Akkumulation von Macht im Lebensmitteleinzelhandel. Als (Unternehmens-)Macht bezeichnet man die Fähigkeit, finanzielle und materielle Ressourcen sowie Humanressourcen zu koordinieren und innerhalb der Wertkette zu steuern (HENDERSON et al. 2002, S. 450). Dieser Macht sind viele kleine Milchbauern mit ihren öko- 
nomischen Strukturen nicht gewachsen. Infolgedessen findet in vielen Ländern Europas ein Hofsterben statt (DriEs et al. 2009; MARQUER 2015). Milch wird stattdessen in einem System produziert, das durch arbeitsteilige und komplexe Strukturen gekennzeichnet ist. Es sind spezialisierte Unternehmen, welche mit großen Viehbeständen Skalenerträge erzielen und neueste Stalltechnologien wie z.B. robotergesteuerte Melktechnik verwenden (CAJA et al. 2016).

Dieser generelle Befund trifft auch auf Slowenien zu. Seit der Unabhängigkeit im Jahr 1991 ist Slowenien durch den Übergang von einer sozialistischen zu einer marktwirtschaftlich geprägten Wirtschaft gekennzeichnet (BOJNEC \& FERTŐ 2014). In diesem Transformationsprozess veränderten sich auch die landwirtschaftlichen Produktionsstrukturen. Die öffentlichen Produktionsbetriebe wurden seit 1992 privatisiert oder aufgelöst. Die Milchproduktion wird heute zur Gänze vom privaten Sektor getragen.

Die Struktur der slowenischen Milchproduktion ist von zahlreichen Kleinbetrieben gekennzeichnet. Aufgrund des überwiegend hügeligen bis hochalpinen Reliefs herrscht in Slowenien starke Konkurrenz um die Nutzung der agrarischen Gunstflächen. Lediglich 23,7\% der Landesfläche sind landwirtschaftlich nutzbar (ZAGORC et al. 2014). GröBere Nutzflächen wie die um Laibach [Ljubljana] oder Cilli [Celje] und die im Dreieck Marburg [Maribor] - Pettau [Ptuj] - Windischfeistritz [Slovenska Bistrica] bilden die Ausnahme. So ist es nicht verwunderlich, dass im Osten des Landes 70\% der Agrargüter produziert werden, denn hier liegen die meisten landwirtschaftlichen Gunstflächen. Der Westen des Landes hingegen ist von Hügeln und Bergen sowie von Karst und mediterranem Klima geprägt. Insbesondere die Almwirtschaft in Form pastoraler Transhumanz ist in den alpinen Regionen weit verbreitet. Die meisten landwirtschaftlichen Betriebe befinden sich in den östlichen Gebieten. Milchbetriebe findet man jedoch überwiegend in den westlichen Regionen. Dem Statistischen Amt Sloweniens zufolge (SURS 2016) sind 10\% aller Betriebe im Haupterwerb milchwirtschaftliche Betriebe. Weitere $8,6 \%$ sind mit der Aufzucht von Schafen und Ziegen beschäftigt, 6\% als Mischbetriebe. So sind auch 48,9\% der agrarisch nutzbaren Flächen Dauergrünlandgebiete (Wiesen und Weiden). Die Milchwirtschaft bildet somit den wichtigsten Teil des slowenischen Agrarsektors (ZAGORC et al. 2014).

Die Qualität der Milch, die in Slowenien produziert wird, entspricht höchsten europäischen Standards. Sie wird durch den Einsatz neuester Techniken seit Jahren kontinuierlich verbessert (VAN Berkum 2009; MaERs 2014). Der überwiegende Teil der Milch wird konventionell produziert, lediglich 1,2\% der Produktion im Jahr 2016 ist biologisch (SURS 2016). Der Anteil biologisch produzierter Milch wächst dennoch stark. Bislang gelten im slowenischen Produktionsprozess andere Qualitätskennzeichnungen als etwa in Österreich. So wird häufig der Ausdruck traditionell verwendet, eine dazu begleitende Zertifizierung fehlt jedoch größtenteils. Weitere Kennzeichnungen wie Silofreiheit oder Heumilch spielen in Slowenien eine untergeordnete Rolle (SCHERMER et al. 2010). Andere Aufwertungsprozesse wie beispielsweise die Weiterverarbeitung sind in Slowenien von größerer Bedeutung.

Die Weiterverarbeitung von Rohmilch wird beinahe ausschließlich von den Molkereien betrieben. Im Jahr 2014 wurden über 86,2\% der Milchproduktion an die Molkereien zur Verarbeitung geliefert (SURS 2015), darunter an die drei großen Unternehmen 
Ljubljanske mlekarne, Celjske mlekarne und Pomurske mlekarne, die zusammen 90\% der slowenischen Milch aufkaufen (Van Berkum 2009; Muminović \& Pavlović 2012).

In den Jahren nach der politischen Wende gab es kaum ausländische Investitionen in den Milchsektor. Dies änderte sich mit der Akquisition von Ljubljanske mlekarne durch die französische Lactalis-Gruppe im Jahr 2012. Allein Ljubljanske mlekarne kaufen ca. 50\% der gesamten produzierten Milch (Muminović \& Pavlović 2012). Die Verteilung von verarbeiteten Milch- und Milchprodukten umfasst über den Verarbeitungssektor hinaus auch private Fachgeschäfte, private Großhändler und die Vertriebsnetze von Einzelhandelsketten. Letztere sind in Slowenien stark konzentriert, sodass bereits 2003 die drei größten Einzelhändler 83\% aller Lebensmittelverkäufe bestritten (DRIES et al. 2009; Kuhar et al. 2014; Statista GmbH 2016a, b).

Im Fokus der einschlägigen Literatur stehen insbesondere Analysen, die Probleme und Chancen aufzeigen, die aus der Einbindung in höherwertige Netzwerke (z.B. GuLATI et al. 2007; MAKONi et al. 2014) oder dem Anschluss an Produktionsnetzwerke resultieren (z.B. Markelova et al. 2009; Dries et al. 2009). Diese Beiträge konzentrieren sich jedoch vor allem auf die Rolle internationaler Leitfirmen und die Veränderungen der zugrunde liegenden Zulieferstruktur auf den weniger entwickelten Zielmärkten. Andere Arbeiten befassen sich mit neuen Entwicklungspfaden bei der konventionellen Milchproduktion wie der Spezialisierung auf funktionelle Lebensmittel in Industriestaaten (MENRAD 2003; Euromonitor 2010; Vitafoods Europe 2017). Keine Beachtung fanden bisher Veränderungen, die sich auf der betrieblichen Ebene der Produzenten vollziehen. Solche Untersuchungen machen aber qualitativ belegbar, warum und wie Produzenten wertbindende Prozesse am Hof konzentrieren, um nicht aus dem Produktionsprozess auszuscheiden. Slowenien eignet sich dazu besonders als Untersuchungsraum, weil viele Umstrukturierungsprozesse stattfinden, die Anpassungsprozesse der Milchproduzenten in besonderem Maße erforderlich machen.

Ziel des vorliegenden Beitrags ist es, die vielfältigen Prozesse aufzuzeigen, die durch die strukturellen Veränderungen in Slowenien entstehen. Es interessieren uns dabei besonders die unterschiedlichen Strategien, die wegen der hohen Abhängigkeit vom vorherrschenden System entwickelt wurden, um dem stark schwankenden Milchpreis gegenüber elastisch zu sein. Dazu wird zunächst der theoretische Rahmen dargelegt, der dieser Forschung zugrunde liegt. Anschließend wird die Struktur des Produktionssystems erläutert und werden systemrelevante Faktoren sowie die Vertriebsstrukturen in der slowenischen Milchwirtschaft beschrieben. Besondere Beachtung finden dabei die Vertriebsstrategien der Mikrokäsereien. Abschließend werden die Forschungsergebnisse resümiert und wird ein Ausblick auf zukünftige Entwicklungen in der slowenischen Milchwirtschaft geboten.

Der Artikel ist Teil einer 2015 durchgeführten qualitativen Erhebung zu neuen Distributionsformen von Mikrokäsereien in Slowenien und veranschaulicht die reziproken Prozesse zwischen einem globalisierten Sektor und den regionalökonomischen Effekten. Insgesamt wurden 22 Tiefen- und Experteninterviews mit verschiedenen Akteuren im slowenischen Produktionssystem von Milch und Milchprodukten durchgeführt und mittels qualitativer Inhaltsanalyse analysiert und interpretiert. Unter den Befragten waren 
Milchbauern, Vertreter von Gebietskörperschaften und des Landwirtschaftsministeriums, Schüler von Bauernschulen, Konsumenten sowie Einzelhändler, die nach ihrer Stellung im Produktionsnetzwerk ausgewählt wurden. Andere Interviewpartner wurden durch ein Schneeballauswahlverfahren gewonnen.

Die Feldforschung konzentrierte sich auf Akteure der Angebots- und Nachfrageseite in der Hauptstadt Laibach sowie in den Statistischen Regionen Oberkrain [Gorenjska statistična regija] und Zentralslowenien [Osrednjeslovenska statistična regija]. Außerdem wurden Akteure in der östlichen Statistischen Region Draugebiet [Podravska statistična regija] befragt, die eine ländliche Käserei betreiben oder dies vorhaben. Zudem wurden neben weiterführender Literatur auch sekundärstatistische Quellen analysiert wie die des Statistischen Amtes von Slowenien.

\section{Theorie: Wertketten in der Milchwirtschaft}

Sozio-ökonomische Prozesse des Wandels lassen sich im zeitgenössischen Kontext besonders aus der Perspektive einer Dienstleistung oder eines physischen Gutes anschaulich nachzeichnen und erklären. Der entscheidende Vorteil dabei ist, dass sektorenübergreifende Prozesse in der Betrachtung einer Wertkettenanalyse berücksichtigt werden (Sturgeon et al. 2008). Netzwerkansätze, seien sie linear (z.B. Global Value Chains) oder netzwerkartig (z.B. Global Production Networks), sind ein geeigneter analytischer Rahmen, um spezifisches Wissen zur Segmentierung und Fragmentierung von arbeitsteiligen Produktionssystemen zu generieren (Henderson et al. 2002; GerefFi et al. 2005; CoE et al. 2008). Dabei werden ein Produkt oder eine Dienstleistung ins Zentrum der Betrachtung gestellt. Davon ausgehend können unterschiedliche vor- und nachgelagerte Prozesse sowie zwischenbetriebliche Beziehungen bei der Produktion von Gütern und Dienstleistungen analysiert werden (CoE et al. 2004). Die globale Perspektive ist bei diesen Ansätzen lediglich die höchstdimensionale Betrachtungsebene, es können aber auch regionale Prozesse mit diesem Rahmen analysiert werden (Henderson et al. 2002; Coe et al. 2004).

Von besonderer Bedeutung für diese analytische Vorgehensweise sind Machtkonstellationen zwischen verschiedenen Akteuren im Produktionssystem, welche in der Regel asymmetrisch sind (GEREFFI et al. 2005). Die Machtverteilung innerhalb eines Netzwerkes ist keinesfalls konstant, sondern verändert sich dynamisch, z.B. durch technische Innovationen, Investitionen oder Institutionen (HENDERSON et al. 2002).

Das Produktionssystem von Lebensmitteln ist ebenfalls von Machtasymmetrien geprägt und wird von großen Lebensmitteleinzelhändlern und großen Zulieferbetrieben dominiert. Oligopole im Lebensmitteleinzelhandel in vielen Ländern Europas ermöglichen es den Akteuren im Einzelhandel, den Wettbewerbsdruck auf die Zulieferer zu übertragen (Hendrickson et al. 2001; Coe \& Hess 2005; Coe \& Wrigley 2007; Reardon et al. 2009; Milberg 2008). Insbesondere im Lebensmitteleinzelhandel führte diese Entwicklung auch zur Etablierung von großen und potenten Zulieferern (COE \& WRIGLEY 2007). Diese Konsolidierung bei den Zulieferern mündet in eine Struktur, die es ihnen ermöglicht, den Wettbewerbsdruck auf Rohwarenhersteller zu übertragen. 
Dieser Preisdruck, der durch die Akkumulation von Macht durch die Lebensmitteleinzelhändler entsteht, betrifft auch das Produktionssystem von Milch und Milchprodukten. Dabei wird das Produktionsnetzwerk von Milch immer mehr globalisiert (CLARK et al. 2007; Swinnen et al. 2006). Dies bezieht sich auf die räumliche Herkunft der Produktionsinputs (z.B. Futtermittel aus Südamerika, Melktechnik aus Italien) wie auch auf das räumliche Muster der Vertriebsregionen, z.B. der europäischen Milchwirtschaft (z.B. Milchpulver nach China) (OECD 2016; Douphrate et al. 2013). Innovationen in den Bereichen Futtermittel, Produktionstechnik und Weiterverarbeitung wirken sich daher zunehmend auf alle globalen Produktionsräume der industriellen Milchwirtschaft und deren Wettbewerbsfähigkeit gleichzeitig aus (Dries et al. 2009). Die Bedingungen für die Wettbewerbsfähigkeit von Betrieben und Produktionsregionen sind immer mehr von naturräumlichen Produktionsfaktoren entkoppelt. Stattdessen sind wirtschaftliche und soziale Faktoren wie der Zugang zu neuem Produktionswissen, die Verfügbarkeit von Fachkräften oder der Zugang zu Märkten von größerer Bedeutung (CLARK et al. 2007; Dairy Australia 2014; Douphrate et al. 2013; OECD 2016).

Investitionen in Betriebe und der Einsatz von innovativen Produktionstechniken können zu Aufwertungsprozessen auf verschiedenen Ebenen führen. Prozessupgrades wie der Einsatz von robotergesteuerten Elementen in der Produktion führen zu kürzeren Arbeitszeiten. Somit können die Produzenten entweder größere Kapazitäten bedienen oder andere Arbeit verrichten. Eine Aufwertung der Produktion bewirkt auch der Einsatz von Hochleistungsrinderrassen wie der Rasse Holstein-Friesian. Deren höhere durchschnittliche Milchleistung resultiert in einer höheren Produktion und somit in weiteren Erlösen (CLARK et al. 2007; VANDEHAR et al. 2016).

Rohmilch ist ein multifunktionaler Lieferant von Kohlehydraten, Proteinen und verschiedenen Vitaminen. Deshalb werden aus dem Rohstoff Rohmilch verschiedene Produkte hergestellt. Die Weiterverarbeitung von Rohmilch zu Trinkmilch ist bereits ein wichtiger wertschöpfender Prozess. Rohmilch darf vielerorts nicht unbehandelt in den Handel gelangen, was u.a. an der hohen Keimbelastung liegt. Pasteurisieren und Homogenisieren sind die grundsätzlichen Verfahren, um Milch vertreiben zu können. Ein weiterer wertschöpfender Prozess besteht in der Weiterverarbeitung der Rohmilch zu Milchprodukten (Produktupgrade) wie Labkäsesorten und Sauermilchprodukten. Ein aktueller Trend ist der massive Ausbau der Produktion von Molkereiprodukten, wodurch zusätzliche Gewinne erzielt werden können, sowie die Entwicklung neuartiger Produkte wie laktosefreier Milch für Menschen mit Laktoseintoleranz oder bestimmter Joghurt-Kulturen, welche die Verdauung anregen sollen (MENRAD 2003). Belege für Aufwertungsprozesse entlang der gesamten Wertkette sind geschützte Herkunftsangaben und Angaben zur Produktionsweise (Bio, Demeter). Weitere wertschöpfende Arbeitsschritte auf Produzentenebene sind die ausschließliche Heufütterung und die daraus entstehende Heumilch.

Die in produktiven Regionen ansässigen Molkereiunternehmen sind in den vergangenen Jahrzehnten zu globalen Unternehmen aufgestiegen (HENDRICKSON et al. 2001). Die Weiterverarbeitung von Milch und deren Distribution ist Ausgangspunkt für Preisbildungsmechanismen und im Wesentlichen auch verantwortlich für die Konzentration von Macht entlang der Wertkette. Bei der Restrukturierung spielen private Unternehmen 
eine wichtige Rolle, so z.B. die oben beschriebenen Akteure aus dem Lebensmitteleinzelhandel oder aber globale Molkereiunternehmen.

Auch wenn aller Wahrscheinlichkeit nach ein Großteil der weltweit produzierten Milch einen informellen Weg geht, sind Verarbeitung und Vertrieb der formal erfassten Milch global organisiert und befinden sich in der Hand einiger großer kapitalintensiver Unternehmen (20 Unternehmen = Marktanteil von 31,4\%) (IFCN 2013). Die Warenströme sind somit stark kanalisiert. Molkereien genießen aufgrund ihrer Bindegliedfunktion zwischen Produktion und Distribution einen privilegierten Zugang zum Absatzmarkt und sind in der Lage, große Milchmengen an sich zu binden. Dies vergrößert ihre Angebotsmacht. Der Großteil der Macht konzentriert sich also am Ende der Wertkette (ABDULSAMAD \& GEREFFI 2016).

Wie die Macht in einem Wertschöpfungsnetzwerk verteilt ist, richtet sich außerdem nach Akteursbeziehungen. Maßgebend dafür sind zum Teil unterschiedliche Unternehmensorientierungen und entsprechende Fähigkeiten, über die die Molkereien verfügen müssen. AbDulsamad \& GerefFi (2016, S. 14) unterscheiden drei Fähigkeiten, die eine Molkerei in Abhängigkeit von ihrer Strategieorientierung besitzt: „(i) a 'trust-based' relationship with milk suppliers: (ii) the reach and scope of their distribution networks; and (iii) innovation and branding“. Demnach besteht ein enger Zusammenhang zwischen dem Kostenführerschaftswillen der Leitfirma - in diesem Fall einer Molkerei und einem starken Vertrauensverlust auf Seiten der ihr zuliefernden Rohmilchproduzenten. Dies trifft zumindest dann zu, wenn eine Molkerei eine produktorientierte Strategie wählt. Weitere Strategien können prozess- und marktorientiert und mit spezifischen, mehr oder weniger gut quantifizierbaren Fähigkeiten verbunden sein (vgl. ABDULSAMAD \& Gerefri 2016). Es zeigt sich also eine Diskrepanz zwischen Produktion und Distribution, die sich zwar je nach Produktionssystem durch die Unternehmensorientierung der Leitfirma unterscheidet. Im Kern jedoch gibt es vielfältige Prozesse, die für Wertschaffung und Wertbindung grundlegend sind.

Die fehlende Weiterverarbeitung durch die Rohmilchproduzenten führt zu einer Verlagerung des Preisdrucks. Der Preisdruck kann beinahe ungehindert an die Produzenten weitergeben werden (Swinnen et al. 2006; Swinnen \& VAndeplas 2011). Dies führt wiederum dazu, dass für die Produzenten wichtige Gewinne verloren gehen und es entweder zur Betriebsaufgabe oder zum Ausbau der Produktionskapazitäten kommt. Hinzu kommen stetig wechselnde politische Rahmenbedingungen. Staatliche Interventionen führten bisher dazu, dass eine fragmentierte Produktionsbasis erhalten blieb. Nicht zuletzt aufgrund der unzähligen Handelsbarrieren und der Vielzahl an Fördermitteln gilt der Milchsektor weiterhin als verzerrt (BEGHIN 2006). Neuere Gesetze sowie Aufhebungen von Regelungen führen zum erneut wachsenden Druck auf die Erzeuger.

Eine Möglichkeit, sich vom behindernden Muster der Produktion zu befreien, ist die Aneignung eigener Weiterverarbeitungsschritte und der Aufbau eigener Distributionswege. Diese können dazu beitragen, dass die zuvor benannten Fähigkeiten von den Molkereien auf die Produzenten verlagert werden und so auch eine Verlagerung der Macht zugunsten der Produzenten eintritt. 


\section{Die slowenische Milchwirtschaft: Struktur, Politik und Regulierung}

Starken Einfluss auf die strukturelle Entwicklung der Landwirtschaft in Slowenien haben politische Regulierungen auf lokaler, nationaler und supranationaler Ebene. Die Milchwirtschaft zählt zu den am stärksten regulierten landwirtschaftlichen Marktsektoren innerhalb der Europäischen Union (EU) und ihrer Mitgliedsstaaten. Ziel der supranationalen und staatlichen Interventionen ist es, die Milchpreise zu stabilisieren und somit die Wettbewerbsfähigkeit der milchproduzierenden Betriebe zu steigern (KEANE \& O'CONNOR 2009; European Union 2014). Bauern erhalten in der Regel Direktzahlungen. So wird beispielsweise die Erhaltung des Dauergrünlandes zusätzlich prämiert. Die europäische Milchwirtschaft ist jedoch tendenziell von einer Überproduktion geprägt. Mit einer Produktionsquotenregulierung wurde in der Vergangenheit versucht, dieser Überproduktion zu begegnen. Damit sollte ein Marktpreisverfall durch ein überhöhtes Angebot in Relation zur Nachfrage verhindert werden. Nach Abschaffung dieser Produktionsquotenregelung im Jahr 2015 wirken nun die Marktpreismechanismen ungehindert. Dies führt zu einem Überangebot und zu einem Preisverfall für die Produzenten (im Jahr 2016 21,62 Euro pro $100 \mathrm{~kg}$ Rohmilch) (EuRopean Commission 2017).

Dieses Überangebot hat eine enorme Machtakkumulation bei Akteuren auf der Nachfrageseite zur Folge und führt zeitweise zu Marktpreisen unter den Erzeugerpreisen. In der Folge kann dieses Überangebot zu Prozessen der Marktkonsolidierung führen, in der landwirtschaftliche Betriebe mangels Betriebskostendeckung zur Aufgabe gezwungen werden. Diese Konsolidierung auf der Angebotsseite wird auch bei Betrachtung der strukturellen Entwicklung der milchproduzierenden Betriebe in Slowenien deutlich (Table 1).

\begin{tabular}{|l|r|r|r|r|r|r|r|r|r|r|r|}
\hline & $\mathbf{2 0 0 5}$ & $\mathbf{2 0 0 6}$ & $\mathbf{2 0 0 7}$ & $\mathbf{2 0 0 8}$ & $\mathbf{2 0 0 9}$ & $\mathbf{2 0 1 0}$ & $\mathbf{2 0 1 1}$ & $\mathbf{2 0 1 2}$ & $\mathbf{2 0 1 3}$ & $\mathbf{2 0 1 4}$ & $\mathbf{2 0 1 5}$ \\
\hline$>500 \mathrm{t} / \mathrm{J}$ & 33 & 35 & 49 & 49 & 53 & 55 & 63 & 73 & 71 & 87 & 102 \\
\hline $100-500 \mathrm{t}$ & 1.180 & 1.224 & 1.343 & 1.346 & 1.334 & 1.364 & 1.409 & 1.452 & 1.363 & 1.424 & 1.483 \\
\hline $50-100 \mathrm{t}$ & 1.748 & 1.723 & 1.699 & 1.683 & 1.637 & 1.601 & 1.555 & 1.539 & 1.480 & 1.431 & 1.424 \\
\hline $10-50 \mathrm{t}$ & 5.062 & 4.720 & 4.292 & 4.053 & 3.792 & 3.517 & 3.320 & 3.077 & 3.025 & 2.800 & 2.594 \\
\hline$<10 \mathrm{t}$ & 1.674 & 1.337 & 1.106 & 914 & 878 & 752 & 606 & 543 & 503 & 478 & 404 \\
\hline Insgesamt & 9.697 & 9.039 & 8.489 & 8.045 & 7.694 & 7.289 & 6.953 & 6.684 & 6.442 & 6.220 & 6.007 \\
\hline
\end{tabular}

Quelle: SURS 2015

Tab. 1: Strukturelle Entwicklung der milchproduzierenden Betriebe in Slowenien. Anzahl der Betriebe 2005-2015

Tabelle 1 stellt die strukturelle Entwicklung der milchproduzierenden Landwirtschaft in Slowenien dar. Im Zeitraum von zehn Jahren (2005-2015) hat sich die Anzahl der Milchbauern von 9.697 auf 6.007 reduziert. Diese Konsolidierung hat vor allem kleinere und mittelgroße Betriebe getroffen. Der Anteil der Betriebe mit geringer Produktions- 
menge von Rohmilch sinkt kontinuierlich. Das Marktsegment der Kleinbauern mit einem Produktionsvolumen von unter zehn Tonnen Milch jährlich hat sich im Zeitraum von zehn Jahren mehr als gedrittelt. Von insgesamt 1.674 Betrieben im Jahr 2005 waren 2015 nur noch 404 Betriebe aktiv. Auch im Marktsegment der Bauern mit 10 bis 50 Tonnen jährlicher Milchproduktion gab es viele Betriebsaufgaben. Insgesamt haben von 5.062 Betrieben im Jahr 2005 mehr als 2.468 Betriebe im Verlauf von zehn Jahren die Milchproduktion eingestellt. Bei den Großbetrieben mit einer Produktion von 100 bis 500 Tonnen und über 500 Tonnen jährlich gab es hingegen im gleichen Zeitraum eine Zunahme der Betriebe. Bei den Milchproduzenten im Spektrum von 100 bis 500 Tonnen stiegen die Betriebszahlen von 1.180 auf 1.483, während die Zahl der Betriebe mit einem Produktionsvolumen von über 500 Tonnen massiv stieg und sich verdreifachte. Somit wird deutlich, dass es zu einer Verschiebung der Produktion im Privatsektor von der Klein- zur Großproduktion kommt. Es droht die Marginalisierung der Gruppe der Kleinstproduzenten.

Die Gründe für diese Entwicklung liegen bei den hohen (pagatorischen) Kosten für die Milchproduktion. Hinzu kommen Kosten für die Erfüllung von Milchhygienestandards, die durch den Gesetzgeber vorgegeben werden. Die Zahl der Akteure sinkt weiterhin durch die Verlagerung zu Gunststandorten. Aufgrund gesetzlicher baulicher Einschränkungen bei der Erweiterung von Stallungen können damit verbundene Investitionen nicht getätigt werden. Mittelgroße und große Betriebe hingegen haben deutlich besseren Zugang zu Investitionsquellen. Diese Betriebe investieren in den Ausbau ihrer Produktionskapazitäten. Damit steigen manche Produzenten zu Großproduzenten auf. Diese wiederum erhalten durch die Kapazitätserweiterungen die Möglichkeit, Skalenerträge zu erzielen. Die Rationalisierung der Produktion führt darüber hinaus zu geringeren Personalkosten bei solchen Produzenten. Verglichen mit großen Produzenten ist das Marktsegment der kleinen und mittleren Betriebe zudem stärker von Betriebsaufgaben durch Generationenwechsel betroffen. Geringe Verdienstmöglichkeiten, schwere körperliche Arbeit und eine ungewisse Zukunft führen zu einem Scheitern der Betriebsübergabe an die nächste Generation.

Größere Betriebe übernehmen und bewirtschaften dann die Flächen der aufgegebenen Kleinbetriebe. Somit sank die Zahl der produzierenden Betriebe, während die Produktionsmenge in den Jahren 2005-2014 nahezu konstant blieb (SURS 2015). Auffällig ist die Verringerung der Milchproduktionsmenge bei kleinen und mittleren Betrieben. Im Jahr 2005 erzeugten diese Betriebe $(<100$ Tonnen $)$ circa 51\% $(2005=262.278 \mathrm{t} / \mathrm{J}, 2014$ $=185.587 \mathrm{t} / \mathrm{J}$ ) der gesamten Menge. Im Jahr 2014 erreichte diese Gruppe nur noch einen Anteil von 35\% am gesamten Volumen. In starkem Kontrast dazu stehen die Betriebe mit einer jährlichen Milchleistung von über 500 Tonnen. Diese Unternehmen steigerten ihre Produktion von 39.324 Tonnen im Jahr 2005 auf 71.449 Tonnen im Jahr 2014.

Diese Konsolidierungsprozesse in der slowenischen Landwirtschaft während der Dekade 2005-2014 haben trotz der protektionistischen EU-Milchquotenregelung (bis 2015) (European Union 2014) stattgefunden. Dies liegt vor allem am Markt- und Machtgefüge, in welches die slowenische Milchwirtschaft auf verschiedenen räumlichen Ebenen strukturell eingebettet ist. So ist auf globaler Ebene die Milchpreisentwicklung stark von der Nachfrage auf neuen Märkten für Milchprodukte, wie z.B. China, abhängig (FulleR et al. 2006). Zudem haben politische Prozesse wie das Handelsembargo Russlands für 
agrarische Güter aus der EU Einfluss auf die angebotene Milchmenge und somit auf die Marktpreise.

Neben diesen preisbildenden globalen Marktprozessen existieren diverse Dynamiken auf der nationalen Nachfrageseite des Marktes für Milchprodukte. Dazu gehören auch die zunehmende Internationalisierung und Konsolidierung des Lebensmitteleinzelhandels, wie die Übernahme des größten slowenischen Einzelhändlers Mercator durch das kroatische Unternehmen Agrokor. Außerdem gewinnen Discounterketten wie Lidl und Hofer (Aldi Süd) Marktanteile am Gesamtmarkt für Lebensmittel in Slowenien. Im Jahr 2014 waren es 16.7\% (Statista GmbH 2016). Der Marktanteil Hofers beispielsweise wächst enorm. Lag er im Jahr 2010 bei 7\%, so erhöhte er sich bis zum Jahr 2014 auf 9,6\% (StATISтА GмвH 2016). Diese Marktkonsolidierung im Lebensmitteleinzelhandel führt zu einer höheren Marktmacht auf der Nachfrageseite und zu einer Restrukturierung des Zuliefersystems für Milchprodukte.

Dazu gehört auch die zunehmende Internationalisierung der Zulieferstrukturen. Internationale Einzelhändler greifen auf etablierte Zulieferunternehmen zurück, um Skalenerträge im Einkauf und somit potenziell höhere Gewinnmargen zu erzielen. So wird inzwischen der slowenische Käsemarkt zunehmend von ausländischen Diskountern und ausländischen Erzeugern beeinflusst.

\section{Vertriebsstrukturen in der slowenischen Milchwirtschaft}

Abbildung 1 zeigt die Vertriebsstrukturen im slowenischen Produktionssystem für Milch und Milchprodukte. Die Verwendungsarten gliedern sich, dargestellt nach ihrer Bedeutung am Gesamtoutput, in (1) indirekter Vertrieb, (2) Eigenbedarf und (3) direkter Vertrieb.

Allgemein sind die Handlungsspielräume der slowenischen Produzenten stark limitiert, wenn es um die Wahl des Distributionskanals geht. Beinahe alle milchproduzierenden Betriebe sind in Kooperativen oder Genossenschaften organisiert, die die Milch zunächst sammeln, um sie dann mit einer größeren Angebotsmacht an die Molkereien weiterzuverkaufen. Die Genossenschaften befinden sich zumeist im Anteilsbesitz der Bauern. Somit üben die Produzenten kollektiven Einfluss auf den Weiterverkauf durch die Genossenschaft aus. Neben den Produzentengenossenschaften werden die Molkereien auch direkt von den Produzenten bedient. Da die Transportkosten hier eine wichtige Rolle spielen, ist der Lieferradius beschränkt. Für die Rohmilchproduktion ergibt sich daraus ein insgesamt stark regionalisiertes Sammelsystem.

„It doesn't make sense for us to bring our milk to a dairy that is more than $20 \mathrm{ki}$ lometres away, for economic reasons. Here in Ptuj, we still have the cooperative and a dairy around. But there are some areas in Slovenia where the farmers have to drive a lot further to reach either a cooperative or a dairy. A lot of dairies got closed in the last 10 years. " (Interview mit einem Produzenten) 


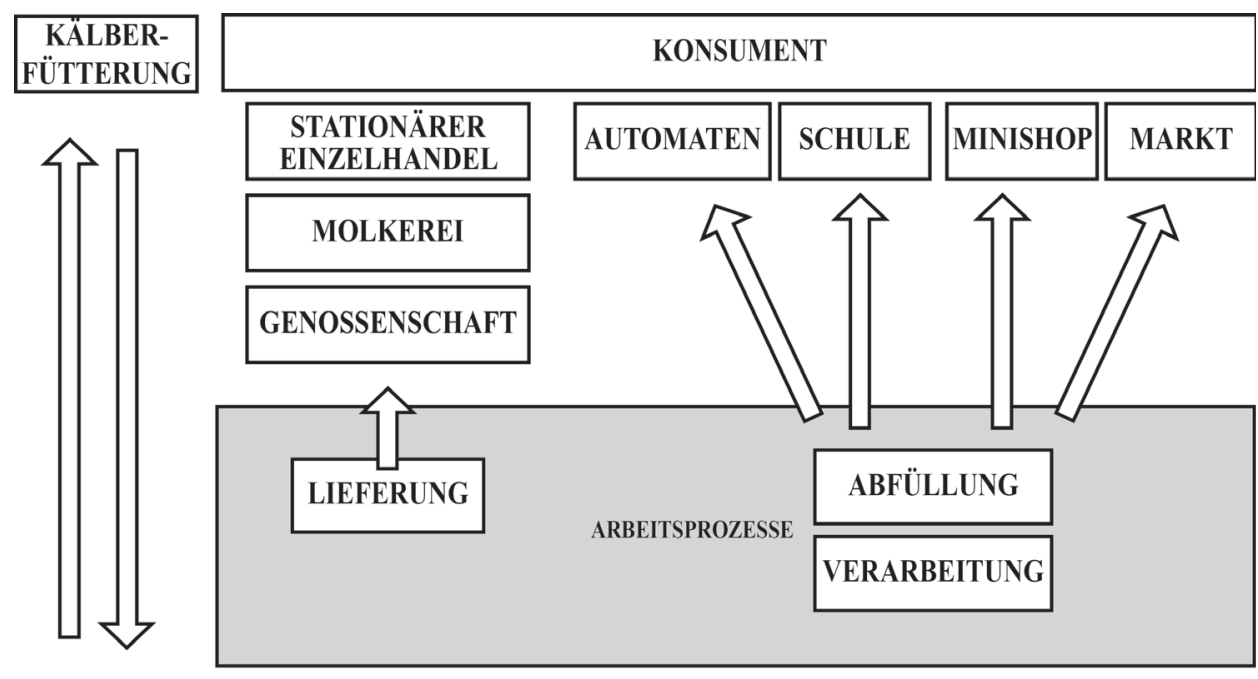

ROHMILCHPRODUKTION

Quelle: Eigene Darstellung

Abb. 1: Vertriebsstrukturen im slowenischen Produktionsnetzwerk

Das Netz der slowenischen Molkereien, welche die Milch zu verschiedenen Produkten verarbeiten, ist bereits stark konsolidiert. Es gibt in Slowenien daher kein dichtes Netzwerk von milchverarbeitenden Betrieben. Große Molkereien befinden sich nur in Regionen, in denen sich auch die Produktion räumlich konzentriert hat. Kleine Molkereien, die sich wie die Mlekarna Planika bei Karfreit [Kobarid] auf Almmilch und Weidemilch für die Käseproduktion spezialisiert haben, gibt es auch an peripheren Standorten wie im abgelegenen Isonzotal [Sočna dolina]. Karfreit liegt zwar nur knapp 90 Kilometer von Laibach entfernt, die Fahrzeit dorthin beträgt jedoch fast zwei Stunden. Somit bedienen die Bauern vornehmlich diese Molkerei.

Milchbauern sind auf das Vorhandensein von Molkereien und Genossenschaften oder Kooperativen angewiesen. So wird am Beispiel der Bohinjska sirarna in der Wochein [Bohinj] deutlich, dass eine kurzzeitige Schließung der Genossenschaft in einer abgelegenen Region auch die Schließung zahlreicher milchproduzierender Betriebe zur Folge haben kann. Das Unternehmen Bohinjska sirarna, das durch Schließung und Wiedereröffnung der Genossenschaft entstand, hat nun Schwierigkeiten, an die für die Herstellung eines traditionellen Käses notwendige Milchmenge zu gelangen.

Im slowenischen Produktionssystem für Milch und Milchprodukte bestehen darüber hinaus weitere direkte Verbindungen zwischen Genossenschaften und Molkereien in Form von Unternehmensbeteiligungen. Molkereien bieten z.B. den Bauerngenossenschaften bei Zahlungsschwierigkeiten Betriebsanteile an. Viele Molkereien sind im Zuge der Finanzund Wirtschaftskrisen (2009/2013) und der daraus resultierenden niedrigen Milchpreise in finanzielle Schieflage geraten. Ein Beispiel hierfür ist die Beteiligung der Genossenschaft 
Mlekarska zadruga in Pettau an der Molkerei Pomurske Mlekarne d.d in Murska Sobota. Die Bauerngenossenschaften bekommen dadurch die Möglichkeit, sich vertikal zu integrieren, was jedoch nicht das Ziel der meisten Genossenschaften ist. Dennoch wird dem Folge geleistet, um akuten Einnahmeausfällen entgegenzuwirken, was wiederum dazu führt, dass die Bauerngenossenschaften bei Missmanagement der Molkereien in Mitleidenschaft gezogen werden können.

Dieses derzeit fragile System trägt den Großteil der slowenischen Distribution. So werden 86,2\% des gesamten Produktionsvolumens über Molkereien und Großeinkäufer abgewickelt (SURS 2015). Der prozentuale Anteil stieg in den Jahren von 2003 bis 2011 von 74,5\% auf insgesamt $87,4 \%$ und erreichte seinen höchsten Stand vor der Eurokrise im Jahr 2012. Die enormen Konzentrationsprozesse dieses Sektors in den vergangenen Jahrzehnten werden daran deutlich. Dabei zeigt die Verwendung der slowenischen Rohmilch ein differenziertes Bild.

\begin{tabular}{|l|r|r|r|r|r|r|r|r|}
\hline & $\mathbf{2 0 0 8}$ & $\mathbf{2 0 0 9}$ & $\mathbf{2 0 1 0}$ & $\mathbf{2 0 1 1}$ & $\mathbf{2 0 1 2}$ & $\mathbf{2 0 1 3}$ & $\mathbf{2 0 1 4}$ & $\mathbf{2 0 1 5}$ \\
\hline $\begin{array}{l}\text { Anlieferung Genossenschaf- } \\
\text { ten \& Molkereien }\end{array}$ & 80,2 & 82,6 & 86,0 & 87,4 & 86,2 & 86,8 & 86,2 & 87,7 \\
\hline Kälberfütterung & 13,5 & 12,2 & 9,4 & 8,2 & 8,9 & 8,4 & 9,1 & 8,0 \\
\hline $\begin{array}{l}\text { Milch zur eigenen Verwen- } \\
\text { dung }\end{array}$ & 3,2 & 2,7 & 2,0 & 1,8 & 2,2 & 2,0 & 2,1 & 1,7 \\
\hline Direktvertrieb Milch & 2,0 & 1,6 & 1,6 & 1,7 & 1,6 & 1,6 & 1,4 & 1,3 \\
\hline Weiterverarbeitung & 1,0 & 0,8 & 1,0 & 1,0 & 1,2 & 1,2 & 1,3 & 1,3 \\
\hline
\end{tabular}

Quelle: SURS 2015

Tab. 2: Verwendung der Rohmilch (in Tonnen) in Slowenien 2008-2015

Der zweitgrößte Wert der in Tabelle 2 dargestellten Rohmilchverwendung entfällt auf den betrieblichen Eigenbedarf. Dieser belief sich auf 11,2\% im Jahr 2014. Dabei wird unterschieden zwischen betrieblichem Eigenbedarf für den Konsum und dem Verbrauch der Rohmilch zur Kälberfütterung. Die Produktion von Milch setzt grundsätzlich eine Laktation voraus, die bei der Kuh nach dem Abkalben einsetzt. Aus diesem Grund muss ein Teil der Milch für die Aufzucht der Kälber verwendet werden. Die Futtermittelproduktion beläuft sich dabei auf 9,1\% der gesamten Produktion. Die verbleibenden 2,1\% werden für den Eigenkonsum verwendet oder werden im Dorf gegen Waren und Dienstleistungen getauscht. Dieser Wert hat sich von 2003 bis 2014 von 17,7\% auf 9,1\%, also um 8,6\% verringert. Die Verringerung dieser Werte, die z.B. durch die Verwendung von Futtermittelersatz zustande kommt, ermöglicht es, dass die Bauern mehr produzierte Milch in den Verkauf fließen lassen können. Das sorgt für höhere Erlöse bei den Milchproduzenten. Die restlichen 2,1\% des Eigenkonsums entfallen auf die Herstellung von Weiterverarbeitungsprodukten für nichtkommerzielle Zwecke.

Eine andere Verwendungsart von Rohmilch ist der Direktvertrieb. Er machte im Jahr 2015 1,3\% aus und hat sich gegenüber 2003 um 1,2\% verringert. Die Weiterverarbeitung 
der Rohmilch zu Milchprodukten stieg im gleichen Zeitraum um 0,3\%, von 1,0\% auf 1,3\%. Grund für den Rückgang des Direktvertriebs sind strengere Anforderungen bei der Lebensmittelhygiene und bei der Eigenvermarktung. Für Slowenien greifen insbesondere die Maßnahmen der Verordnung EG 2004. Der kurze Anstieg im Jahr 2009 ist durch die Wirtschaftskrise zu begründen. In dieser Zeit gerieten viele Molkereien in finanzielle Probleme, die zur Folge hatten, dass die Bauern ihre Milch nur schwer weiterverkaufen konnten. Gleiches gilt für die Eurokrise im Jahr 2012, die viele Staaten des südlichen Europas, darunter auch Slowenien, traf.

Der Direktvertrieb bildet eine wichtige Alternative zu anderen, indirekten Vertriebsformen, insbesondere für kleinere Produzenten. Der Anteil dieser Vertriebsart sinkt aber im Vergleich zu anderen Vertriebsformen. Dies liegt zunächst daran, dass der Output der kleinen Produzenten, die solche Vertriebsarten wählen, sehr gering gegenüber dem Output einiger weniger, dafür sehr großer Produzenten ist, die zu einer indirekten Vertriebsform tendieren. Im Gegensatz zu den Werten des gesamten Outputs entschließen sich jedoch immer mehr slowenische Produzenten zum Direktvertrieb, um Schwankungen im Rohmilchpreis auszugleichen.

Die entscheidende Determinante hierbei ist der Milchpreis. Er wird zwischen Molkereien und dem Einzelhandel verhandelt. Dafür werden Aufträge vom Einzelhandel ausgeschrieben. Molkereien bewerben sich auf diese ausgeschriebenen Aufträge hin. Anschließend wird der Preis in Verhandlungen festgelegt. Diese Verhandlungen wirken sich dann auf den Auszahlungspreis der Molkereien und somit auf die Genossenschaften und die Erzeuger aus. Andere Einflussgrößen auf den Milchpreis sind die Qualität der Rohmilch, die sich am Protein- und Fettgehalt bemisst, sowie die Art der Milch nach Herkunft oder Produktionsweise.

\section{Direkte Distributionsformen}

Zur Differenzierung der Vertriebswege kam es insbesondere ab 2008 und ab 2012. Vor allem von kleinen und mittelgroßen Produzenten wird der Vertrieb direkt organisiert und über verschiedene Kanäle gestaltet. Die häufigsten Vertriebswege sind (1) der Verkaufsraum am Hof des Erzeugers, (2) Verkaufsautomaten, (3) Bauernmärkte sowie (4) die Schulspeisung.

Produktion und Verkauf der Milchprodukte erfolgen oft nebeneinander am Hof des Milcherzeugers. Häufig sind die Käserei und der Verkaufsraum in benachbarten Räumen zu finden. Verkaufsräume oder Minishops sind integrale Bestandteile der Vertriebsorganisation von Mikrokäsereien. Der Verkaufsraum besteht zumeist nur aus einigen wenigen Kühlschränken oder Vitrinen. Um die Attraktivität des Ladens zu erhören, werden saisonal auch weitere Produkte aus der eigenen Produktion verkauft sowie gelegentlich auch fremdbezogene Waren von Familienmitgliedern, Verwandten oder befreundeten Erzeugern wie Obst und Eier sowie verarbeitete Produkte wie Marmeladen und Nudeln. Die 
zusätzlichen Angebote bieten die Möglichkeit, weitere Umsätze und Gewinne zu erzielen. Dies ist gerade angesichts der Investitionskosten nicht unwichtig, denn sie belaufen sich für eine Mikrokäserei durchschnittlichen auf bis zu 30.000 Euro.

„, The average investment for the equipment is about 20,000 - 30,000 Euros. And then we don't talk about constructing a new building or something. It's just the equipment. The adaptation only. (...) Most farms have existing spaces, which they transform. " (Interview mit der Landwirtschaftskammer)

Die Investitionskosten variieren je nach Ausgangssituation. Darüber hinaus müssen andere Kosten berücksichtigt werden, die die baulichen Maßnahmen betreffen. Wenn die Gebäudestruktur es zulässt, werden z.B. für die Käserei und den Verkaufsraum Gebäudeteile wie die Garage umfunktioniert. Wenn das nicht möglich ist, werden neue Gebäude errichtet, was jedoch einen erheblichen finanziellen Mehraufwand für die Produzenten bedeutet. Die Einrichtungskosten sind relativ hoch, vor allem für Kleinstlandwirte mit weniger als 50 Tonnen jährlicher Produktion. Bei gesicherter Hofnachfolge sind Investitionen in Käserei und Verkauf jedoch generationenübergreifend. Daher können sie auch kleine Produzenten in Kauf nehmen.

„, The investment is a huge amount of money for me. But if you take 20 years into account, then it should be OK. If there are children interested in the farm and thinking of taking it over, that is no short-term investment. Then the investment is really long-termed. I am nearly 60 years old and my son goes to an agricultural school. So he is going to take over the farm. That was very important for the investment. " (Interview mit einem Produzenten)

Die Etablierung neuer Geschäftsfelder ist für die slowenischen Milchbauern mit hohen Kosten und Risiken verbunden. Dies betrifft sowohl die Produktion als auch den Verkauf. Sie ergeben sich aus der kurzen Lebensdauer der nicht-pasteurisierten Waren. Daher sind kurze Vertriebswege und Warenketten elementar für die Produkte der Milchbauern. Um die Verfügbarkeit der Produkte zu erhöhen, werden die Verkaufsräume auch außerhalb der definierten Verkaufszeiten für einen Kundentermin geöffnet.

„Before we started our little production of cheese and yoghurt we didn't think about service, but now we have to think about all options to make the most of it. It's true that just selling milk was already a full-time job. Now, we have to work even more. Selling cheese in the minishop is okay for us. At least one of us is always at home. This person can then sell. There is no problem. But we are now enlarging our business through vending machines. This again is more complicated, because we are very busy with the refilling of the machines. " (Interview mit einem Produzenten)

Verkaufsautomaten für Frischkäse und Frischmilch bieten den Produzenten eine weitere Möglichkeit des Direktvertriebs. Die Standorte der Automaten werden strategisch 
gewählt und sind vor allem in Kundennähe in den Städten Sloweniens zu finden. Standorte mit einer hohen Passantenfrequenz, z.B. Tankstellen oder Supermärkte, werden bevorzugt. Mikrokäsereien suchen also nicht nur die räumliche Nähe zum Lebensmitteleinzelhandel, sondern treten mit ihm auch direkt in Konkurrenz. Dies ergibt sich aus der Macht der Einzelhandelsgeschäfte und deren Anziehungskraft auf Kunden. Außerdem werden Automaten bevorzugt an stark frequentierten Aus- oder Einfallsstraßen oder direkt am Hofgelände aufgestellt.

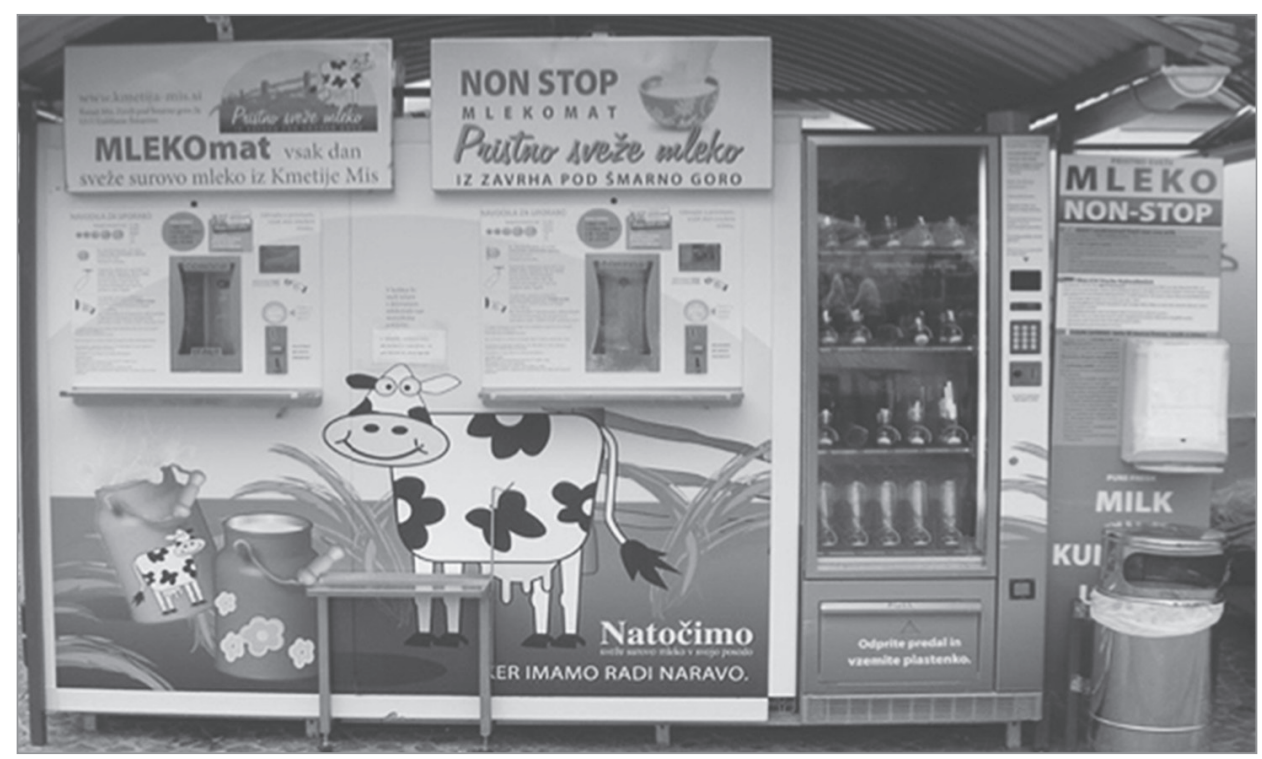

Quelle: eigene Aufnahme

Abb. 2: Milchverkaufsautomat

„We have a location at the daily market in Ljubljana. There, we sell between 100 and 150 litres a day. The amount we sell really depends on the weather. But it is a good place for us. There are a lot of people every day. But we also have to pay 300 Euros per month. At this place we sell very well and a lot of milk. The money for the amount of milk we sell is okay. " (Interview mit einem Produzenten)

Technisch besteht ein Automat (siehe Abb. 2) in der Regel aus einem Milchspender und einem separaten Flaschenspender. Dies ermöglicht es den Kunden, nach Wunsch eigene Gebinde mitzubringen oder direkt am Automaten eine passende PET-Flasche zu beziehen. Je nach Standort können bis zu 150 Liter pro Tag umgesetzt werden. Der Verkaufspreis der Milch liegt bei 70 Cent je Liter, was zum Zeitpunkt der Feldforschung 30 Cent mehr war als z.B. der Ankaufspreis für Rohmilch der Genossenschaft in Pettau. Diese Preisdifferenz erklärt die Profitabilität des Automatengeschäfts trotz der relativ hohen Beschaffungskosten von ca. 2.000 Euro je Automat. Zudem fallen weitere Kosten 
für Unterhalt und Reparatur sowie die Standmiete an. Dennoch bilden Automaten einen für die Produzenten vorteilhaften Distributionsweg, was auch zum Ausbau des Verkaufsautomatennetzes in Slowenien geführt hat. Die sequenzielle Verkürzung der Warenkette und der Aufbau eigener Vertriebsstrukturen erhält ihnen auch die Kontrolle über die Preisgestaltung. Die geschlossenen Strukturen lösen sich dadurch weiter auf.

Lokale Märkte sind ebenfalls ein integraler Bestandteil der Vertriebsorganisation von Mikrokäsereien. Viele Bauern organisieren den Verkauf der Frischwaren auf Märkten selbstständig. Wochen- und Bauernmärkte sind das Rückgrat dieser Verkaufsform. Weitere Formen sind Sondermärkte/Degustationen, Biomärkte, saisonale Märkte und Dorffeste. Märkte sind in der Regel durch eine hohe Passantenfrequenz gekennzeichnet, sodass es auch viele zufällige Kunden gibt. Allerdings führt die Witterungsabhängigkeit zu Schwankungen bei den Verkaufserlösen.

„, The difference compared to before is that we receive episodic money. Delivering to the dairy means a monthly payment for us. Now, we generate the most of our money on a weekend basis. Before, I thought it could be very risky, but the response is okay for us. It could be better, but we believe in a long-term change and that the business will raise during the next years. To lower the risk, we look for all possibilities. We try markets. We try anything at the moment. " (Interview mit einem Produzenten)

Außer der Standmiete fallen bei Märkten keine Mietkosten an. Somit können Fixkosten für Ladenmiete und Personal gering gehalten werden. Die Personalkosten sind zumeist auch deshalb gering, weil der Verkauf in der Regel von der Landwirtsfamilie selbst durchgeführt wird. Dies ermöglicht es auch, den Verkauf mit entsprechendem Produkt- und Herkunftswissen $\mathrm{zu}$ begleiten und $\mathrm{zu}$ fördern. Insbesondere bei überregionalen Märkten wie dem biologischen Erzeugermarkt in Laibach ist dies der Fall.

Die slowenischen Bauern- und Wochenmärkte bilden ein periodisches Netz der Versorgung. Dabei können zwei unterschiedliche Markttypen identifiziert werden, die für die Milchbauern von Bedeutung sind: Im urbaneren Kontext gibt es erstens Wochenmärkte mit permanenten Shops und angebundenem Straßenmarkt und zweitens Wochenmärkte mit ausschließlichem Straßenverkauf. Letztere werden häufig von Mikrokäsereien präferiert. Der wöchentliche Zyklus strukturiert für die Mikrokäsereien nicht nur den Verkauf zeitlich, sondern bietet auch Intervalle zur effektiven Produktion. Da Käserei für viele eine Nebentätigkeit ist, muss die Produktion ohnehin in die sonstigen Tätigkeiten integriert werden. Insbesondere in der Anfangsphase von Mikrokäsereien sind Wochenmärkte eine geeignete Form, um das Produkt zu testen und Erfahrungen zu sammeln.

Etwas Besonderes ist der polyzentrische Markt von Laibach. Er besteht hauptsächlich aus zwei Zentren - einem Innenstadtmarkt und einem am östlichen Stadtrand. Der Innenstadtmarkt hat viele Geschäfte und Marktstände, die Käse, Joghurt und Milch anbieten. Spezialisierte permanente Käsegeschäfte bieten Käsevariationen von zahlreichen, zumeist unterschiedlichen slowenischen Produzenten an, mit denen schon seit vielen Jahren Lieferbeziehungen bestehen. 
„It's hard to enter the specialised cheese shops in the city center. If you produce products, such as yoghurt, it's very unlikely that you sell your products through these shops. You need to produce more middle- and hard-type cheese with very good quality. This could be a next step. But for the moment, if you want to sell your products on your own, then open your own shop. For example, the farm Pustotnik, in Gorenja vas. He has already some shops in Ljubljana. He constantly widens this product range. " (Interview mit einem Produzenten)

Der Markt am Pogačarjev trg ist z.B. ein solcher periodischer biologischer Markt. Er bietet spezialisierten Rohmilchverarbeitern die Möglichkeit, hochpreisige Produkte wie Weidemilch oder biologisch zertifizierte Milch an eine sowohl kaufkraftstarke als auch gebildete Käuferschicht abzusetzen.

„, The customer wants to buy this kind of food, but they don't know where to get it, if not at the market or the supermarket. Now we get calls from people from Ljubljana. "(Interview mit einem Produzenten)

Im Internet werden die Produkte kaum gehandelt, da dort kaum Bestellungen aufgegeben werden können. Das Internet hat lediglich eine Repräsentationsfunktion. Dennoch schätzen viele Produzenten und Kunden die Internetpräsenz zur Kommunikation von Telefonnummern oder Öffnungszeiten. Das Angebot von Internetseiten und Onlineshops wächst daher kontinuierlich, auch wenn die Verderblichkeit der Ware diese Marketingform besonders einschränkt. Da aber nur wenig investiert werden muss, lohnt sich eine eigene Homepage trotzdem.

Eine besondere Form der Onlinepräsenz sind thematische Homepages. Sie haben den Vorteil, dass sich die Produzenten mit wenig Aufwand eintragen können und über ein weiteres thematisches Umfeld oder eine regionale Szene informiert wird. Thematische Homepages können wie im Falle der Wocheiner Käseroute auch Touristen mobilisieren. Auch die Kosten für die Homepage werden geteilt oder vollständig vom Anbieter übernommen, wenn es sich wie im Falle des Verbandes ländlicher Käsereien um eine institutionelle Initiative handelt.

Der Tourismus, besonders der Erholungstourismus, bietet zudem noch weitere Distributionsmöglichkeiten. Wachsender Beliebtheit erfreut sich v.a. der Urlaub am Bauernhof, für den frühere Almwirtschaften besonders beliebte Ziele darstellen. Für die Produzenten bedeutet dies, dass ein Teil der produzierten Rohmilch für den touristischen Betrieb verwendet wird. Die stetig wachsende Zahl an Gästezimmern auf Bauernhöfen sorgt ferner für eine Neuausrichtung vieler landwirtschaftlicher Betriebe. Auf der Internetseite der nationalen Tourismusinitiative „I feel Slovenia“ werden mittlerweile über 300 Bauernhöfe aufgelistet, die Gästezimmer anbieten. Neben dem Urlaub auf dem Bauernhof ist auch die temporäre Einbindung des Gastes in den landwirtschaftlichen Betrieb möglich. Der überwiegende Teil der Angebote bezieht sich auf Milchbetriebe.

Die Einkommensstruktur der Bauernhöfe ändert sich damit in Richtung eines dienstleistungsorientierten Gewerbes, und die slowenischen Milchproduzenten geben ihre Rolle als reine Rohmilchlieferanten zunehmend auf. Neben einer Verkürzung führt dies auch 
zu einer Diversifizierung der Wertkette. Der Tourismus ist für periphere Räume zumeist die einzige Möglichkeit, den unrentablen Hof aufrechtzuerhalten. Die Milchproduzenten profitieren dabei vom naturnahen Image Sloweniens im Ausland, das zu einer Internationalisierung der Konsumenten führt.

Eine weitere und viel praktizierte Form ist die Belieferung öffentlicher Einrichtungen wie von Schulen. Die Schulspeisung gewinnt in Slowenien zunehmend an Bedeutung für kleine und mittlere Milchproduzenten. Sie handeln mit Schulen kurz- bis mittelfristige Verträge aus - in der Regel für ein Schuljahr. Es handelt sich zumeist um mündliche Absprachen über tagesgenaue Lieferungen von Milch und Milchprodukten.

Die Milchproduzenten profitieren vom direkten Vertrieb der Waren und von der Möglichkeit, weiterverarbeitete Produkte zu verkaufen. Allerdings ist der koordinative Aufwand sehr groß und sind die Gewinnmargen an den Produkten vergleichsweise gering. Dies liegt unter anderem daran, dass die Schulen portionierte Verpackungen haben wollen. Diese verkleinern den koordinativen Aufwand der Schulen, erhöhen aber den der Produzenten und verursachen bei diesen Mehrkosten durch Verpackungen und längere Arbeitszeiten.

\begin{abstract}
„Delivering the yoghurts to the schools is problematic because of the individual packaging. The producer must deliver bigger amounts to the schools, but he can't use bigger tubs for that. There still is work to do to get the awareness on a higher level (...). The schools have enough money, even for more expensive yoghurts, say twenty or thirty percent. If they switched to reusable glass, this would be great. But mostly they want individual packaging because they don't want extra labour. " (Interview mit der Landwirtschaftskammer)
\end{abstract}

Potenziell bilden die slowenischen Schulen einen großen Markt für kleine Milchproduzenten. So gab es im Schuljahr 2015/2016 in Slowenien 781 Grundschulen, 182 Mittelschulen und Gymnasien sowie 67 Fachhochschulen und Fachschulen (TAŠTANOSKA 2015). Bisher wurden die Schulen durch große Molkereien beliefert. Doch durch das aktive Engagement von Produzenten kommt es allmählich zu einem Umdenken. Es kann längerfristig auch bewirken, dass Kinder und Eltern wieder bewusst mit lokalen Erzeugnissen in Kontakt treten.

\title{
6 Fazit
}

Am Beispiel der slowenischen Mikrokäsereien wird deutlich, welche Rolle die Verkürzung der Wertkette spielt. Ihre sequenzielle Verkürzung bei gleichzeitiger Diversifizierung führt dazu, dass die erzeugte Milch von den Produzenten zu kostendeckenden Preisen verkauft werden kann. Dies ist eine notwendige Reaktion auf die Tatsache, dass sich die Marktmacht auf der Distributionsseite konzentriert hat. Der dadurch ausgelöste Preisdruck auf die Produzenten (SwINNEN et al. 2006) führte zu einer Marktbereinigung, die vor allem kleine und mittlere Milchbetriebe traf. Die Reaktion der kleinen Produzenten 
besteht in der Entwicklung von Strategien der Direktvermarktung ihrer Produkte. Solche sind die Weiterverarbeitung von Rohmilch zu Milchprodukten und die Schaffung eigener, kurzer Distributionswege. Die Distributionsformen reichen von periodisch stattfindenden Märkten über eigene Verkaufsräume bis zur Aufstellung von Verkaufsautomaten. Oftmals führt die Kombination unterschiedlicher Strategien zum Erfolg. Wichtig ist auch, dass sich der Produzent vom Rohmilchlieferanten zum dienstleistungsorientierten Unternehmen wandelt.

\section{Literaturverzeichnis}

Abdulsamad A., Gereffi G. (2016), Dairy Value Chains in East Africa. - http://www.cggc.duke.edu/ $p d f s$ /09-30-2016_IGC_dairy_report_(final).pdf(Zugriff: 24.04.2016).

BEGHIN J.C. (2006), Evolving dairy markets in Asia: Recent findings and implications. In: Food Policy, 31, 3, S. 195-200.

BOJNEC Š., FERTÖ I. (2014), Export competitiveness of dairy products on global markets: The case of the European Union countries. In: Journal of Dairy Science, 97, 10, S. 6151-6163.

Caja G., Castro-Costa A., Knight C.H. (2016), Engineering to support wellbeing of dairy animals. In: Journal of Dairy Research, 83, 2, S. 136-147.

Clark D.A., Caradus J.R., Monaghan R.M., Sharp P., Thorrold B.S. (2007), Issues and options for future dairy farming in New Zealand. In: New Zealand Journal of Agricultural Research, 50, 2, S. 203-221.

Coe N.M., Dicken P., Hess M. (2008), Global production networks: realizing the potential. In: Journal of Economic Geography, 8, 3, S. 271-295.

Coe N.M., Hess M. (2005), The internationalization of retailing: implications for supply network restructuring in East Asia and Eastern Europe. In: Journal of Economic Geography, 5, 4, S. 449-473.

Coe N.M., Hess M., Yeung H.W.C., Dicken P., Henderson J. (2004), 'Globalizing' regional development: a global production networks perspective. In: Transactions of the Institute of British geographers, 29, 4, S. 468-484.

Coe N.M., Wrigley N. (2007), Host economy impacts of transnational retail: the research agenda. In: Journal of Economic Geography, 7, 4, S. 341-371.

Dairy Australia (Hrsg.) (2014), Australian Dairy Industry. Dairy Australia. - http://www.dairyaustralia.com.au/Industry-information/About.aspx (Zugriff: 10.08.2016).

Douphrate D.I., Hagevoort G.R., Nonnenmann M.W., Lunner Kolstrup C., Reynolds S.J., Jakoв M., Kinsel, M. (2013), The Dairy Industry: A Brief Description of Production Practices, Trends, and Farm Characeristics Around the World. In: Journal of Agromedicine, 18, 3, S. 187-197.

Dries L., Germenji E., Noev N., Swinnen J.F. (2009), Farmers, vertical coordination, and the restructuring of dairy supply chains in Central and Eastern Europe. In: World Development, 37, 11, S. 1742-1758.

Euromonitor International (Hrgs.) (2010), Diverse Corporate Strategies to Exploit Opportunities in Global Dairy. - http://www.euromonitor.com (Zugriff: 10.08.2016).

European Commission (Hrsg.) (2017), Milk Market Observatory. - https://ec.europa.eu/agriculture/ sites/agriculture/files/market-observatory/milk/pdf/eu-raw-milk-prices_en.pdf (Zugriff: 12.03.2017). 
European Union (Hrsg.) (2014), Smooth Phasing-Out of the Milk Quotas in the EU. - http://cor. europa.eu/en/documentation/studies/Documents/milk-quotas-eu.pdf(Zugriff: 18.11.2016).

Fuller F., Huang J., Ma H., Rozelle S., (2006), Got milk? The rapid rise of China's dairy sector and its future prospects. In: Food Policy, 31, 3, S. 201-215.

Gereffi G., Humphrey J., Sturgeon T. (2005), The governance of global value chains. In: Review of International Political Economy, 12, 1, S. 78-104.

Gulati A., Minot N., Delgado C., Bora S. (2007), Growth in high-value agriculture in Asia and the emergence of vertical links with farmers. In: European Union, Committee of the Regions (Hrsg.), Global supply chains: standards and the poor: how the globalization of food systems and standards affects rural development and poverty, S. 98-108. - http://cor.europa.eu/en/ documentation/studies/Documents/milk-quotas-eu.pdf(Zugriff: 16.10.2016).

Henderson J., Dicken P., Hess M., Coe N., Yeung H.W.C. (2002), Global production networks and the analysis of economic development. In: Review of International Political Economy, 9, 3, S. 436-464.

Hendrickson M., Hefrernan W.D., Howard P.H., Hefrernan J.B. (2001), Consolidation in food retailing and dairy. In: British Food Journal, 103, 10, S. 715-728.

International Farm Comparison Network (IFCN) (2013), n.d. Marktanteile der führenden Hersteller von Molkereierzeugnissen weltweit im Jahr 2013. - https://de.statista.com/statistik/ daten/studie/326114/umfrage/marktanteile-der-fuehrenden-hersteller-von-molkereierzeugnissen-weltweit (Zugriff: 19.04.2017).

Keane M., O'Connor D. (2009), Price volatility in the EU dairy industry: causes, consequences and coping mechanisms. Report prepared for the European Dairy Association.

Kuhar A., ErJavec E., Borovšak K. (2014), Restructuring of the Slovenian food industry in the pre- and post-EU accession period. In: Agroeconomia Croatica, 4, 1, S. 40-49.

Makoni N., Redda T., Van der Lee J., Mwai R., Van der ZijpP A. (2014), White gold: Opportunities for dairy sector development collaboration in East Africa. Wageningen, Centre for Development Innovation.

Markelova H., Meinzen-Dick R., Hellin J., Dohrn S. (2009), Collective action for smallholder market access. In: Food Policy, 34, 1, S. 1-7.

Marquer P. (2015), Historical data on the milk sector (1983-2013). In: Agriculture and fisheries statistics in focus, 4. - http://ec.europa.eu/eurostat/statistics-explained/index.php?title $=A g$ riculture,forestry_and_fishery_statistics\&stable $=0 \&$ redirect $=$ no (Zugriff: 15.07.2016).

Menrad K. (2003), Market and marketing of functional food in Europe. In: Journal of Food Engineering, 56, 2, S. 181-188.

Milberg W. (2008), Shifting sources and uses of profits: Sustaining US financialization with global value chains. In: Economy and Society, 37, 3, S. 420-451.

Ministry of Agriculture and Environment of the Republic of Slovenia (MAERS), Agricultural Institute of Slovenia (Hrsg.) (2014), Poročilo o stanju kmetijstva, živilstva, gozdarstva in ribištva v letu 2013. Pregled po kmetijskih trgih [Bericht über die Situation von Landwirtschaft, Ernährung, Forstwirtschaft und Fischerei im Jahr 2013. Erhebung über Agrarmärkte]. - http://www.mkgp.gov.si/fileadmin/mkgp.gov.si/pageuploads/podrocja/ ZP_2013_trgi.pdf (Zugriff: 05.05.2017).

Muminović S., PAVlović V. (2012), Profitability of dairy industry in Slovenia, Croatia and Serbia. In: Mljekarstvo, 62, 2, S. 96-110.

Organisation for Economic Co-operation and Development (OECD) (Hrsg.) (2016), Dairy and Dairy Products. In: OECD-FAO Agricultural Outlook 2016-2025, S. 110-129. Paris, OECD Publishing. 
Reardon T., Barrett C.B., Berdegue J.A., Swinnen J.F.M. (2009), Agrifood Industry Transformation and Small Farmers in Developing Countries. In: World Development, 37, 11, S. 1717-1727.

Schermer M., Matscher A., Borec A. (2010), The perception of quality aspects for mountain products in long supply chains - Cases from Slovenia and Austria. In: BOKU-University of Natural Resources and Applied Life Sciences (Hrsg.), Building sustainable rural futures: the added value of systems approaches in times of change and uncertainty. $9^{\text {th }}$ European IFSA Symposium, Vienna, Austria, 4-7 July 2010, S. 1588-1598, Wien, BOKU - University of Natural Resources and Applied Life Sciences.

TAŠTANOSKA T. (Hrsg.) (2015), The education system in the Republic of Slovenia. Ljubljana, Ministry of Education, Science and Sports of the Republic of Slovenia.

Statista GmbH (Hrsg.) (2016a) n.d. Marktanteil von Aldi im Lebensmitteleinzelhandel in Europa nach Ländern in den Jahren 2010 und 2014. - https://de.statista.com/statistik/daten/studie/457005/umfrage/marktanteil-von-aldi-in-europa-nach-laendern/ (Zugriff: 16.11.2016).

Statista GmbH (Hrsg.) (2016b) n.d. Marktanteil von Lidl im Lebensmitteleinzelhandel in Europa nach Ländern in den Jahren 2010 und 2014. - https://de.statista.com/statistik/daten/studie/457036/umfrage/marktanteil-von-lidl-in-europa-nach-laendern/ (Zugriff: 16.11.2016).

Statistični urad Republike Slovenite (SURS), Agency of the Republic of Slovenia for Agricultural Markets and Rural Development (Hrsg.) (2015), Annual production and utilization of milk on agricultural holdings: Cows' Milk On Agricultural Holdings 2015.xls, Slovenia, 2014. - http://www.stat.si/StatWeb/en/News/Index/6156 (Zugriff: 20.06.2016).

Statistični urad Republike Slovenije (SURS), Agency of the Republic of Slovenia for Agricultural Markets and Rural Development (Hrsg.) (2016). - http://pxweb.stat.si/pxweb/ Dialog/varval.asp? ma $=1516501 E \& t i=\&$ path $=. /$ Database/Environment $/ 15$ agriculture fishing/03_agricultural_holdings/01_15165_land_use/\&lang=1 (Zugriff: 20.06.2016).

Sturgeon T., Van Biesebroeck J., Gereffi G. (2008), Value chains, networks and clusters: reframing the global automotive industry. In: Journal of Economic Geography, 8, 3, S. 297-321.

Swinnen J.F.M., Dries L., Noev N., GermenjI E. (2006), Foreign Investment, Supermarkets, and the Restructuring of Supply Chains: Evidence from Eastern European Dairy Sectors (= LICOS discussion paper 165). Leuven, LICOS-KULeuven.

SWINNEN J., VANDEPLAS A. (2011), Rich consumers and poor producers: quality and rent distribution in global value chains. In: Journal of Globalization and Development, 2, 2, S. 1-30.

Van Berkum S. (2009), An Assessment of the Competitiveness of the Dairy supply Chain in New Member States, Candidate and Potential Candidate Countries. In: AgriPolicy, Enlargement Network for Agripolicy Analysis. - http://www.euroqualityfiles.net/AgriPolicy/Report\%20 2.1/AgriPolicy\%20Synthesis\%20report\%20Dairy\%20Chain\%20Analysis\%20May\%20 2009.pdf (Zugriff: 10.04.2016).

Vandehaar M., Armentano L., Weigel K., Spurlock D., Tempelman R., Veerkamp R. (2016), Harnessing the Genetics of the Modern Dairy Cow to Continue Improvements in Feed Efficiency. In: Journal of Dairy Science, 99, 6, S. 4941-4954.

VitAFoods EuRope (Hrsg.) (2017), Market size of fortified/functional foods worldwide in 2014 and 2020 (in billion U.S. dollars). - https://www.statista.com/statistics/252803/global-functional-food-sales/ (Zugriff: 16.12.2016).

Weiss W. (2007), Regionalität und regionale Lebensmittel. Vienna, Springer.

Zagorc B., MolJK B., Pintar M. (2014), Poročilo o stanju kmetijstva, živilstva, gozdarstva in ribištva v letu [Bericht über die Situation der Landwirtschaft, Ernährung, Forstwirtschaft und Fischerei im Jahr]. Ljubljana, Kmetijski inštitut Slovenije (KIS). 